\title{
A facile amino-functionalization of poly(2-oxazoline)s' distal end through sequential azido end-capping and Staudinger reactions
}

Shigehito Osawa ${ }^{1,2}$, Takehiko Ishii ${ }^{3}$, Hiroyasu Takemoto ${ }^{4}$, Kensuke Osada ${ }^{* 3,5}$, and

Kazunori Kataoka*1,2,6,7

${ }^{1}$ Innovation Center of NanoMedicine (iCONM), Kawasaki Institute of Industrial

Promotion, 3-25-14, Tonomachi, Kawasaki-ku, Kawasaki, 210-0821, Japan.

${ }^{2}$ Department of Materials Engineering, Graduate School of Engineering, The University

of Tokyo, 7-3-1 Hongo, Bunkyo, Tokyo 113-8656, Japan.

${ }^{3}$ Department of Bioengineering, Graduate School of Engineering, The University of

Tokyo, 7-3-1 Hongo, Bunkyo, Tokyo 113-8656, Japan.

${ }^{4}$ Laboratory for Chemistry and Life Science, Institute of Innovation Research, Tokyo 
Institute of Technology, R1-11, 4259 Nagatsuka, Midori-ku, Yokohama 226-8503, Japan.

${ }^{5}$ Japan Science and Technology Agency, PRESTO, 4-1-8 Motomachi, Kawaguchi,

Saitama 332-0012, Japan.

${ }^{6}$ Division of Clinical Biotechnology, Center for Disease Biology and Integrative

Medicine, Graduate School of Medicine, The University of Tokyo, 7-3-1 Hongo,

Bunkyo, Tokyo 113-0033, Japan.

${ }^{7}$ Policy Alternatives Research Institute, The University of Tokyo, 7-3-1 Hongo, Bunkyo,

Tokyo 113-0033, Japan.

*Corresponding authors: Professor Kensuke Osada and Professor Kazunori

\section{Kataoka}

E-mail: osada@bmw.t.u-tokyo.ac.jp (K. O.), kataoka@ bmw.t.u-tokyo.ac.jp (K.

K.)

KEYWORDS

poly(2-oxazoline), amino end-functionalization, azido termination, amino conversion,

Staudinger reaction 


\section{Abstract}

Facile and quantitative method to introduce primary-amino group into the distal chain end of poly(2-substituted 2-oxazoline)s (POx)s, which have attracted progressive interest as platform polymers for various bio-functionality materials, was established here via two-step reactions; First, termination of cationic polymerization of 2-oxazoline using sodium azide as the end-capping reagent, followed by the conversion of the azido group to an amino group using triphenylphosphine (TPP) by the Staudinger reaction. The azido introduction step and the subsequent amino conversion step was accomplished within 1 hour and 3 hours, respectively, to ultimately obtain $\omega$-amino-poly(2-ethyl-2-oxazoline) (PEtOx- $\left.\mathrm{NH}_{2}\right)$ with $96 \%$ functionality. The synthesized PEtOx- $\mathrm{NH}_{2}$ was subjected to ring-opening polymerization of $N^{\varepsilon}$-trifluoroacetyl-L-lysine $N$-carboxy anhydride (Lys(TFA)-NCA) to successfully obtain the block copolymer PEtOx- $b$-PLys(TFA), which can be utilized as a platform polymer to develop polymer therapeutics, thus, demonstrating the versatility of the presently reported procedure for further functionalization of POx derivatives. 


\section{Introduction}

Poly(2-substituted 2-oxazoline) (POx) obtained by living cationic

ring-opening polymerization of the corresponding oxazoline monomer, has recently

attracted significant attention as a class of functionality polymers, particularly for

biomedical applications [1-3]. The substituent on the 2-position of the oxazoline unit

gives POxs a variety of functionalities. For example, poly(2-methyl 2-oxazoline)

(PMeOx) and poly(2-ethyl-2-oxazoline) (PEtOx) are the hydrophilic and biocompatible

polymers approved by the U.S. Food and Drug Administration [3,4]. In addition to this,

POxs with substituents such as n-propyl (PnPrOx) and isopropyl (PiPrOx) have thermo-responsive properties, so that they can exhibit lower critical solution temperature (LCST) in the range of physiological condition $[1-3,5,6]$. It is also feasible to integrate additional functionalities to POxs by introducing various functional groups, such as amino group [7], alkenyl and alkynyl groups [8], thiol group [9], sugars [10], and so forth, to the side chain of POxs by preparing appropriate monomers with these functional groups or their precursors. The living nature of oxazoline polymerization is 
also an appealing point. POxs with quite a narrow molecular weight distribution, $M_{\mathrm{w}} / M_{\mathrm{n}}$ as small as 1.01 measured by MALDI-TOF MS, can easily be obtained under optimized conditions [6]. These unique features promote the use of POxs in various biomedical applications, such as surface coating [11,12], bioconjugation $[13,14,15]$, hydrogels $[16,17]$, and drug/gene carriers $[4,18,19]$.

One of the key issues to further expand the utilities of POxs in the afore-mentioned applications is the development of a facile and quantitative procedure to introduce reactive groups selectively at their chain end for conjugation, tethering, and polymerization chemistry. Particularly, introduction of a primary amino group is attractive considering its wide reactivity. It should be noted that primary amine can initiate polymerization of $N$-carboxyl anhydride (NCA) to produce POx- $b$-poly(amino acid) block copolymers, which may be feasible alternatives to poly(ethylene glycol) (PEG)-b-poly(amino acid) block copolymers widely utilized in drug delivery formulations [20,21]. A few procedures have been reported to introduce a primary amino group at the end of POxs. J. S. Park et al and L. Tauhardt, et al reported a procedure to introduce phthalimide, followed by conversion to an amino group [22,23]. 
M. Meyer et. al demonstrated a procedure to introduce 4-( $N$-Boc-amino)-piperidine, followed by deprotection of the Boc group to obtain POx-piperidine- $\mathrm{NH}_{2}$ [24]. Despite these procedures being able to introduce a primary amino group at the $\omega$-chain end of POxs, it takes a prolonged time period for the reaction to complete.

Here, we have developed a facile and quantitative procedure to introduce a primary amino group into the $\omega$-chain end of POxs: Terminating the living cationic polymerization of oxazolines by the end-capping with sodium azide to obtain azido-terminated POx as the first step [25,26], followed by the Staudinger reaction using Triphenylphosphine (TPP) to convert the azido group to a primary amino group $[27,28]$. This procedure was completed within several hours, and a high conversion of $96 \%$ functionality was obtained. Furthermore, to demonstrate the utility of amino-functionalized POxs for further applications, we conducted a ring-opening polymerization of L-lysine(TFA)-NCA from the synthesized $\omega$-amino-poly(2-ethyl-2-oxazoline) $\quad\left(\mathrm{PEtOx}_{-} \mathrm{NH}_{2}\right)$ to obtain PEtOx-b-poly(L-lysine(TFA)) with a narrow molecular weight distribution, which is expected to be useful as a platform polymer for drug and gene delivery applications. 


\section{Materials and Methods}

\subsection{Materials}

2-Ethyl-2-oxazoline (EtOx) (Tokyo Kasei Kogyo Co., Ltd., Tokyo, Japan),

2-n-propyl-2-oxazoline (nPrOx) (Tokyo Kasei Kogyo Co., Ltd., Tokyo, Japan), acetonitrile (Wako Pure Chemical Industry, Osaka, Japan), and dimethyl sulfoxide (DMSO) (Wako Pure Chemical Industry, Osaka, Japan) were used after distillation over calcium hydride. Methyl p-toluenesulfonate (Tokyo Kasei Kogyo Co., Ltd., Tokyo, Japan) was used after distillation over phosphorus pentoxide. Sodium azide (Wako Pure Chemical industry, Osaka, Japan), triphenylphosphine (TPP) (Tokyo Kasei Kogyo Co., Ltd., Tokyo, Japan), and other reagents were used as received. $N^{\varepsilon}$-trifluoroacetyl-L-lysine $N$-carboxy anhydride (Lys(TFA)-NCA) was prepared by the Fuchs-Farthing method [29].

\subsection{Synthesis of azido-terminated PEtOx}


Synthesis of azido-terminated PEtOx $\left(\mathrm{PEtOx}^{-N_{3}}\right)$ is outlined in Scheme 1a.

The polymerization of EtOx was carried out according to the literature methods $[6,19,22]$. Briefly, an initiator of methyl $p$-toluenesulfonate $(62 \mathrm{mg}, 0.66 \mathrm{mmol})$ was dissolved in $9 \mathrm{~mL}$ of acetonitrile. This was followed by the addition of EtOx ( $3.4 \mathrm{~g}, 34$ mmol) to the solution. The mixture was then stirred at $42{ }^{\circ} \mathrm{C}$ for 96 hours. A small portion of the reaction solution was sampled to evaluate the progress of polymerization using MALDI-TOF-MS (Bruker ultrafleXtreme, Bruker Daltonics, Bremen, Germany). After confirming the polymer formation, $\mathrm{NaN}_{3}(430 \mathrm{mg}, 6.6 \mathrm{mmol})$ was added and stirred at $70{ }^{\circ} \mathrm{C}$ for 24 hours to terminate the polymerization [25,26]. All procedures were carried out under an argon atmosphere. After the reaction, the mixture was filtered to remove unreacted $\mathrm{NaN}_{3}$. This was followed by dialysis with deionized water for five times. The solution was then lyophilized to recover the product as a white powder. The obtained polymer was characterized by SEC (GPC TOSOH HLC8220 system with RI detector) equipped with G4000HHR and G3000HHR (TOSOH, Tokyo, Japan) columns using DMF containing $10 \mathrm{mM} \mathrm{LiCl}$ as an eluent $(0.8 \mathrm{~mL} / \mathrm{min})$. Matrix Assisted Laser Desorption/Ionization Time-of-Flight mass spectrometry (MALDI-TOF MS) and 
infrared (IR) spectroscopy (IRA-1, JASCO, Tokyo, Japan) were also utilized for the characterization. In MALDI-TOF measurement, $\alpha$ cyano-4-hydroxycinnamic acid was used as a matrix and potassium trifluoroacetate was used to form potassium adduct ion of the sample.

\subsection{Conversion of azido-terminated PEtOx (PEtOx- $\left.\mathrm{N}_{3}\right)$ to amino-terminated}

\section{PEtOx (PEtOx-NH2)}

The azido terminus of PEtOx was converted into a primary amino-end by a Staudinger reaction with TPP (Scheme 1 (b)). PEtOx-N 3 (440 mg, $0.10 \mathrm{mmol}$ ) was dissolved in $20 \mathrm{~mL}$ of methanol, followed by the addition of TPP (520 mg, $2.0 \mathrm{mmol})$. The solution was stirred at $40{ }^{\circ} \mathrm{C}$ for 24 hours to convert the azido group to iminophosphazido group. Subsequently, deionized water $(40 \mathrm{~mL})$ was added to the solution to hydrolyze the iminophosphazido group to yield an amino group. The unreacted TPP was removed by filtration. After evaporation of the solvent under reduced pressure, the residue was re-dissolved in $20 \mathrm{~mL}$ of deionized water and filtered again. The filtrate was lyophilized to obtain a white powder. MALDI-TOF MS was 
utilized to evaluate the progress of azide-amine conversion. In MALDI-TOF measurement, 1,8,9-trihydroxyanthracene was used as matrix and potassium trifluoroacetate was used to form the potassium adduct ion of the sample. The product was also analyzed by ion-exchange HPLC, equipped with a refractive index (RI) detector and a cation-exchange column (TOSOH TSKgel SP-5PW), using $2 \mathrm{mM}$ phosphate buffer (PB) (pH 6.5) as an eluent. The conversion ratio of the amine was determined from the area ratio of the peaks corresponding to the sample with (Elution volume: $\sim 6.2 \mathrm{~mL}$ ) and without (Elution volume: $\sim 2.7 \mathrm{~mL}$ ) $\omega$-amino group.

\subsection{Optimization of the reaction time for conversion of $\mathrm{PEtOx}-\mathrm{N}_{3}$ to $\mathrm{PEtOx}-\mathrm{NH}_{2}$}

The reaction time for the conversion of $\mathrm{PEtOx}-\mathrm{N}_{3}$ to $\mathrm{PEtOx}-\mathrm{NH}_{2}$ was estimated as follows: PEtOx-N $3(440 \mathrm{mg}, 0.10 \mathrm{mmol})$ was dissolved in $20 \mathrm{~mL}$ of methanol at $40{ }^{\circ} \mathrm{C}$, followed by the addition of TPP $(520 \mathrm{mg}, 2.0 \mathrm{mmol})$ to initiate the reaction. $2 \mathrm{~mL}$ Aliquots were collected from the reaction solution at 1, 3, 6, 15, and 24 hours after the initiation, and was added to $4 \mathrm{~mL}$ of water to convert the $\omega$-end of the PEtOx to an amino group. After collecting the product according to the procedure 
described in Section 2.3, the azido-to-amine conversion ratio was determined by ion-exchange HPLC.

\subsection{Optimization of the reaction time for terminating EtOx polymerization using}

$\mathrm{NaN}_{3}$.

The reaction time required for azido termination was estimated from the amino functionality of the final $\mathrm{PEtOx}-\mathrm{NH}_{2}$ products. Firstly, EtOx was polymerized for 96 hours in the same manner described above. Excess $\mathrm{NaN}_{3}$ (10 equivalents of the initiator) was added to the solution to terminate polymerization and the mixture was stirred at $70^{\circ} \mathrm{C}$. After $1,3,6,9,12$, and 24 hours from the initiation of the reaction, an aliquot $(1 \mathrm{~mL})$ was collected from the reaction solution and treated as described in Section 2.2 to obtain PEtOx-N3. Each sample $(20 \mathrm{mg})$ was then dissolved in $1 \mathrm{~mL}$ of methanol and reacted with an excess amount of TPP $(20 \mathrm{mg}, 20$ equivalents of azido group in PEtOx-N 3 ) at $40{ }^{\circ} \mathrm{C}$ for 3 hours, followed by the addition of water to afford the conversion of iminophosphazido group to amino group. After purification as described in Section 2.3, the amino functionality of the final product was determined using 
ion-exchange HPLC.

\subsection{Synthesis of PEtOx-b-PLys(TFA) initiated from PEtOx- $-\mathrm{NH}_{2}$}

Lys(TFA)-NCA (180 mg, $0.67 \mathrm{~mol})$ was dissolved in $2.0 \mathrm{~mL}$ of DMF and was added to a DMF (2.0 mL) solution of PEtOx-NH2 $(44 \mathrm{mg}, 0.010$ mol), which was prepared according to the procedures describing in Section 2.2 2.3, and stirred at $25{ }^{\circ} \mathrm{C}$ for 72 hours under an argon atmosphere. The mixture was precipitated using $100 \mathrm{~mL}$ of diethyl ether. The product was filtered and dried under vacuum. The synthesized PEtOx- $b$-PLys(TFA) was analyzed by size exclusion chromatography (SEC) and ${ }^{1} \mathrm{H}-\mathrm{NMR}$ spectroscopy (JEOL ECS 400, JEOL, Tokyo, Japan). The degree of polymerization of PLys(TFA) was determined by ${ }^{1} \mathrm{H}-\mathrm{NMR}$ by comparing the peak areas of the $\beta$-, $\gamma$-, and $\delta$-methylene protons (- $\left.\underline{\mathrm{CH}}_{2} \mathrm{C}_{2} \underline{\mathrm{C}}_{2} \underline{\mathrm{CH}}_{2} \mathrm{NH}_{3}, \delta=1.30-1.90 \mathrm{ppm}\right)$ of the side chain of the Lys unit to the methyl protons of the side chain of PEtOx $\left(-\mathrm{NCOCH}_{2} \mathrm{C}_{3}, \delta=0.83-0.90 \mathrm{ppm}\right)$. 


\section{Results and Discussion}

\subsection{Synthesis of amino-functionalized PEtOx}

Scheme 1 illustrates the reaction to introduce a primary amino group onto the $\omega$-chain end of PEtOx by means of cationic ring-opening polymerization of EtOx using methyl $p$-toluenesulfonate as an initiator. Polymerization was carried out in acetonitrile at $42{ }^{\circ} \mathrm{C}$ for 96 hours according to the previous procedure $[6,19,22]$. This condition was selected to avoid possible side reactions such as chain transfer and chain coupling, which may occur when the reaction is conducted at a higher temperature $[6,22]$. The molecular weight of PEtOx in the aliquot sampled from the reaction mixture was confirmed to be 4,500 Da by MALDI-TOF MS. Subsequently, $\mathrm{NaN}_{3}$ was added to the reaction mixture as the end-capping reagent to introduce azido group at the $\omega$-chain end of the prepared PEtOx [25]. The reaction was continued for 24 hours at an elevated temperature, $70{ }^{\circ} \mathrm{C}$, to increase the solubility of $\mathrm{NaN}_{3}$ in acetonitrile. The polymer sample that was isolated from the reaction solution was confirmed to be unimodal with a narrow molecular weight distribution $\left(M_{\mathrm{w}} / M_{\mathrm{n}}=1.06\right)$ as revealed from the SEC data shown in Figure 1a, demonstrating the living nature of EtOx polymerization without the 
occurrence of side reactions. The MALDI-TOF MS also indicated successful polymerization with unimodal and narrow molecular weight distribution $\left(M_{\mathrm{w}} / M_{\mathrm{n}}=\right.$ 1.01) (Figure 1b). The mass difference between a series of major signals was consistent with the mass of the repeating unit of the polymer $(=99.13)$ in MALDI-TOF MS analysis, further supporting the successful formation of PEtOx (Figure 1b). In addition, the vibrational peak corresponding to the azido group was clearly observed at $2100 \mathrm{~cm}^{-1}$ in the IR spectrum, indicating the introduction of azido group into PEtOx [25]. Furthermore, MALDI-TOF MS showed that the mass of the major signals corresponded to the molecular weight of the potassium adduct of $\mathrm{CH}_{3}-\mathrm{PEtOx}-\mathrm{N}$, a form of two nitrogen atoms detached from the azido group. Note that this detachment is known to occur due to the high laser power of the MALDI-TOF mass spectrometer [30,31]. The occurrence of weak signals to the left hand side of the major signals $\left(\mathrm{CH}_{3}-\mathrm{PEtOx}-\mathrm{N}-\mathrm{K}^{+}\right)$ may reflect the presence of a small fraction of polymer chains initiated by protons of $p$-toluenesulfonic acid as a hydrolytic byproduct of the methyl $p$-tosylate initiator [22], as each mass difference $(\mathrm{m} / \mathrm{z}=14.05)$ agreed with the mass difference between $\mathrm{CH}_{3}-\mathrm{PEtOx}-\mathrm{N}-\mathrm{K}^{+}$and $\mathrm{H}-\mathrm{PEtOx}-\mathrm{N}-\mathrm{K}^{+}(=14.03)$. Consequently, it is safe to conclude 
that the azido group was quantitatively introduced at the $\omega$-chain end of PEtOx under these experimental conditions.

(a)

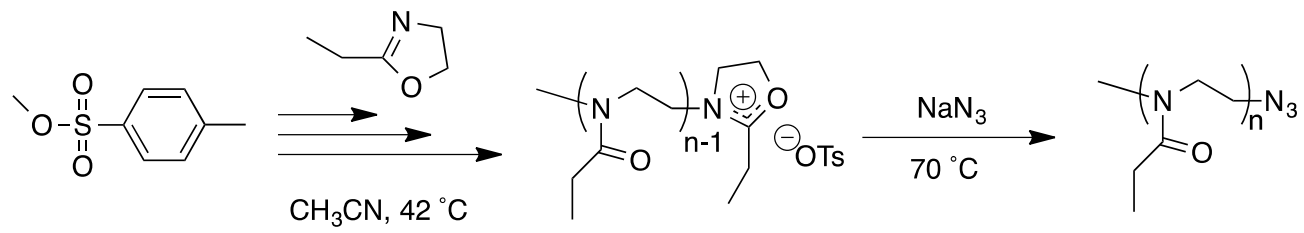

(b)

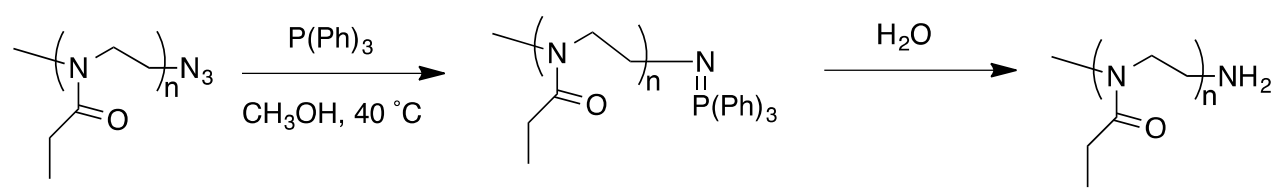

Scheme 1. Synthetic route to obtain PEtOx- $\mathrm{NH}_{2}$. (a) Polymerization of EtOx followed

by a termination reaction with $\mathrm{NaN}_{3}$. (b) Conversion of the azido group to the amino group by one-pot sequential reactions with TPP (Staudinger reaction) and water. 

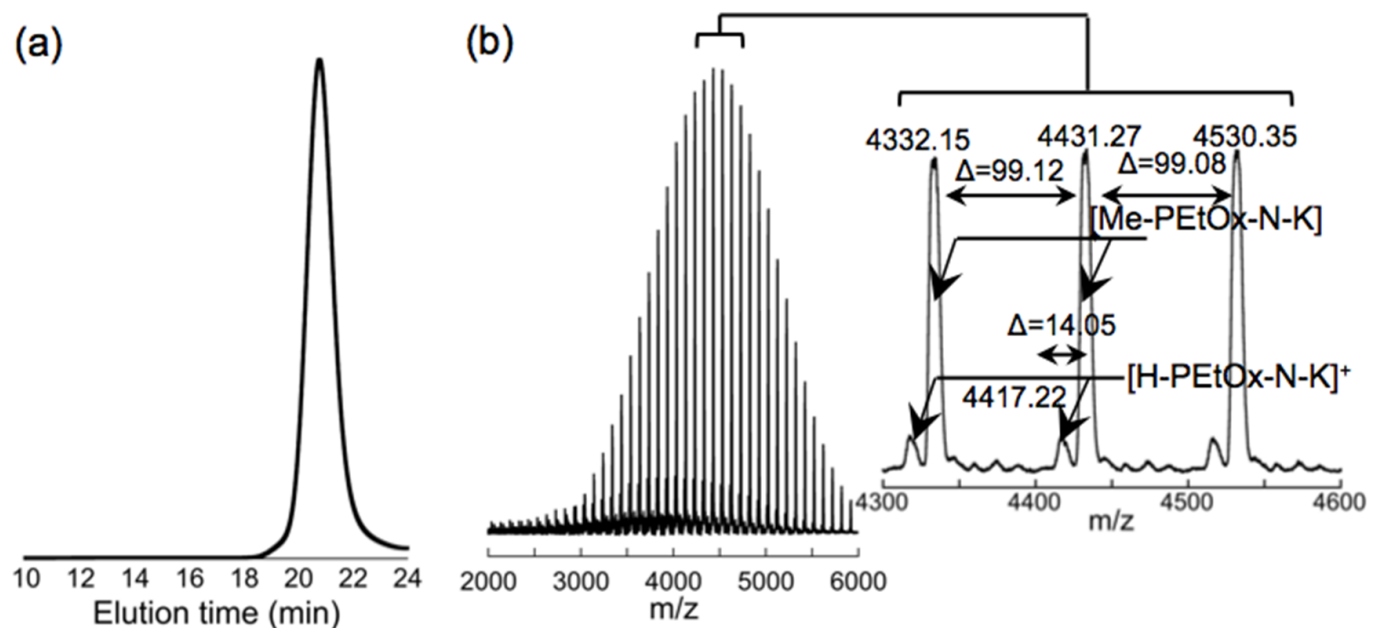

(c)
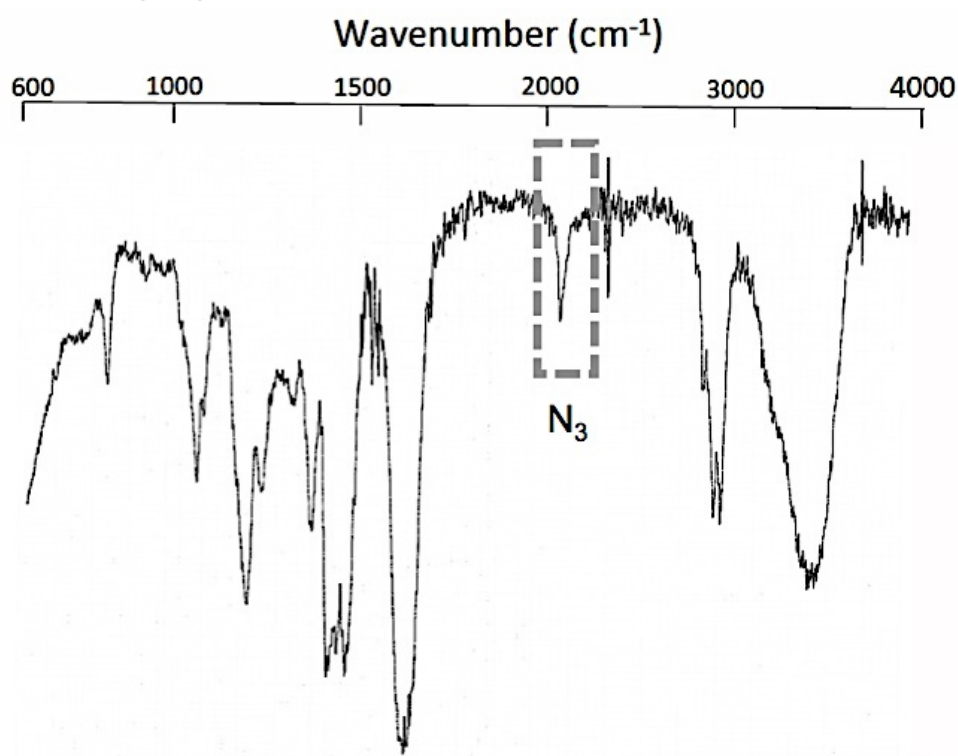

Figure 1. Characterization of the synthesized PEtOx-N3 by (a) SEC, (b) MALDI-TOF

MS (left) with a magnified region of 4300-4600 m/z (right), and (c) IR measurements.

Subsequently, the introduced azido terminus of PEtOx was converted into a primary amino group by the Staudinger reaction using TPP $[27,28]$. PEtOx-N 3 was 
reacted with TPP for 24 hours to convert the azido group to an iminophosphazido group, followed by the addition of water to obtain $\mathrm{PEtOx}-\mathrm{NH}_{2}$ by hydrolysis. Note that the hydrolysis reaction was completed instantaneously to yield the amino group. The Staudinger reaction had negligible effect on the molecular weight distribution of the polymer as the SEC profile of the sample after the reaction was similar with that of before (Figure S1). This was further confirmed by the similar profile of the MALDI-TOF MS spectra for the samples before and after the reaction (Figure 2). These MALDI-TOF MS spectra were analyzed to evaluate the progress of the Staudinger reaction. The MALDI-TOF MS measurement for the azido-terminated PEtOx, which was detected as Me-PEtOx-N-K ${ }^{+}$in the MS, gave a series of peaks reflecting the distribution in the polymerization degree as shown in Figure 2(a). Each peak further split into the subpeak of fractions with different composition of isotopes as shown in the expanded panel in Figure 2(a). Among these subpeaks, the leftmost one is the monoisotopic fraction that only contains the principal isotope of each element. Then, as indicated in Figure 2(a), the major MS peaks (No. 1-6) of Me-PEtOx-N-K ${ }^{+}$were selected, and the measured mass values of their monoisotopic fraction were listed in 
Table 1. By using the atomic mass of each principal isotope $\left({ }^{12} \mathrm{C}=12.000,{ }^{1} \mathrm{H}=1.0078\right.$, $\left.{ }^{16} \mathrm{O}=15.995,{ }^{14} \mathrm{~N}=14.003,{ }^{39} \mathrm{~K}=38.964\right)$, the corresponding polymerization degree, and accordingly, the molecular weight of each monoisotopic fraction were calculated. As seen in Table 1, the calculated molecular weight had good agreement with the measured mass values of the monoisotopic fraction in peak No. 1 to 6 , supporting the formation of the assigned structure. The same procedure was applied for the sample after the Staudinger reaction, which is detected as Me-PEtOx- $\mathrm{NH}_{2}-\mathrm{K}^{+}$in MS (Figure 2(b)). The monoisotopic fractions of selected peaks with the definite polymerization degree (No. 1-6), corresponding to the set of the peaks in the MS of Me-PEtOx-N-K ${ }^{+}$ (Figure 2(a)), gave the measured mass values in good agreement with the calculated molecular weights (Table 2). There was observed $1.97 \sim 2.03(\mathrm{~m} / \mathrm{z})$ increase in the mass value of monoisotopic fraction for all of the corresponding peaks after the Staudinger reaction (Table 2). Apparently, this increment agrees to the expected mass change (= $2 \mathrm{H}$ ) from the original Me-PEtOx-N-K ${ }^{+}$to Me-PEtOx-NH $2-\mathrm{K}^{+}$, indicating successful progress of the Staudinger reaction. Consequently, the conversion ratio of azide to amino group was determined by ion-exchange HPLC equipped with a cation-exchange 
column. Here, PB ( $\mathrm{pH}$ 6.5) was used as the elution buffer to facilitate electrostatic interaction with the cation-exchange column by promoting protonation of the amino group of the product. The HPLC chart presented minor and major peaks at elution volumes of $2.7 \mathrm{~mL}$ and $6.2 \mathrm{~mL}$, respectively (Figure 3). The minor peak could be a non-amino functionalized fraction, which can be assigned to the unreacted fraction because the elution volume corresponds to that of the original PEtOx- $\mathrm{N}_{3}$ (Figure 3 dashed line), or possibly a fraction of hydroxyl end-capped PEtOx produced due to contaminated water. The major peak was then assigned to a fraction of amino-converted PEtOx $\left(\mathrm{PEtOx}_{-} \mathrm{NH}_{3}{ }^{+}\right)$. The conversion ratio to the amino-group was calculated to be $96 \%$ from the area ratio of these two peaks, demonstrating the high efficiency of this procedure to introduce a primary amino group into the $\omega$-chain end of PEtOx. The same amino-functionalization ratio of $96 \%$ was also achieved for $\mathrm{PnPrOx}$, indicating the versatility of this procedure for any other POx derivatives (Figure S2). 
(a)
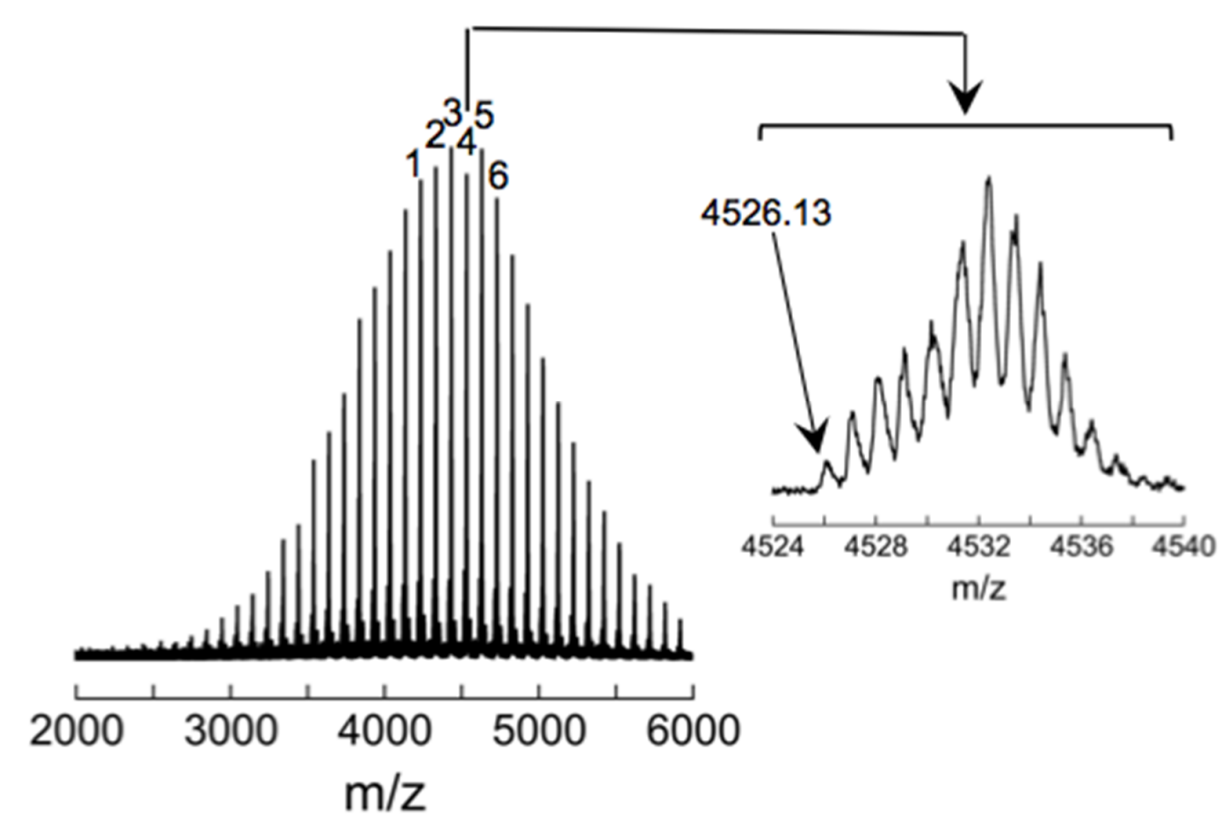

(b)

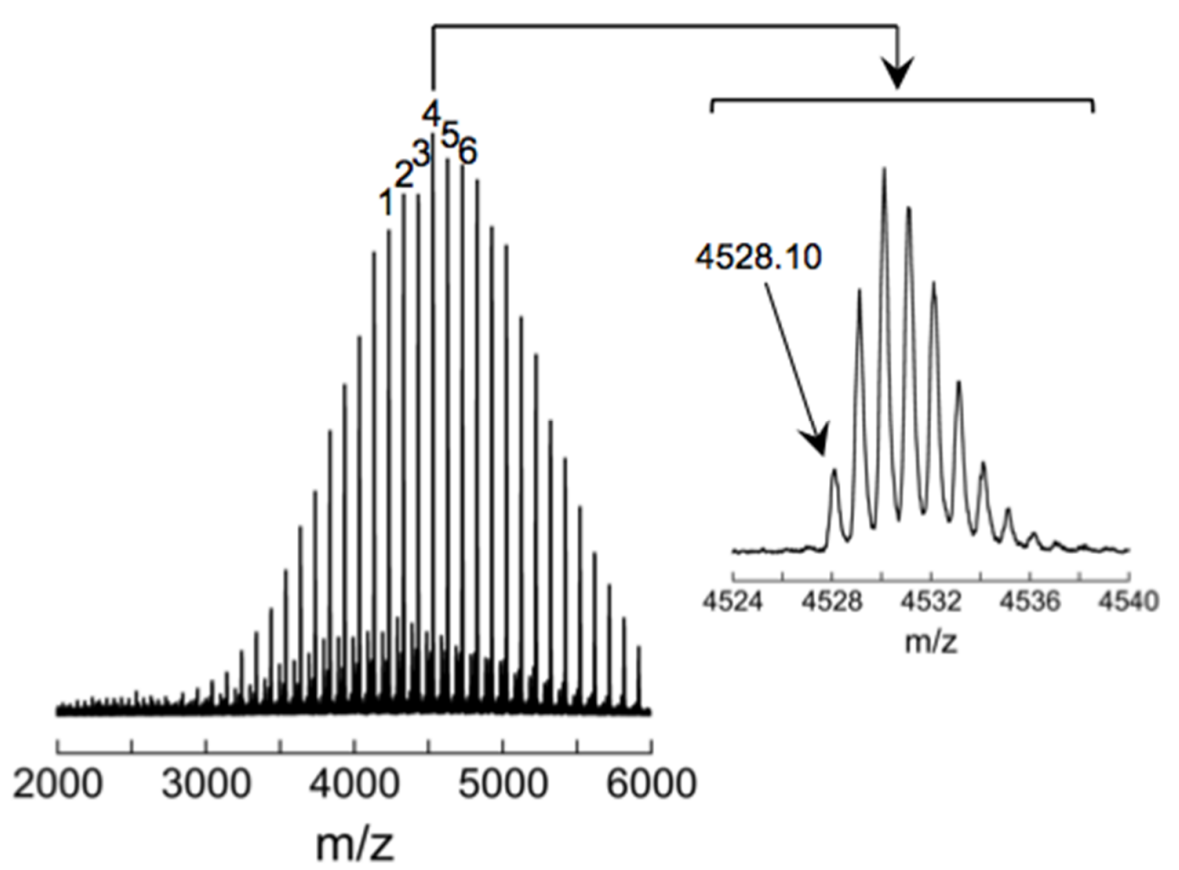

Figure 2. MALDI-TOF MS spectra for the samples before (a) and after (b) the Staudinger reaction. Peak No. 4 in the spectra was expanded to observe the signals from 
the fractions with different composition of isotopes. The leftmost peak corresponds to the signal from the monoisotopic fraction.

Table 1. Mass values of monoisotopic fraction in the selected peaks of MALDI-TOF MS spectra of Me-PEtOx-N-K

\begin{tabular}{cccc}
\hline Peak number ${ }^{(\mathrm{i})}$ & $\begin{array}{c}\text { Measured mass } \\
\text { value of the } \\
\text { monoisotopic } \\
\text { fraction (m/z) }\end{array}$ & $\begin{array}{c}\text { Calculated } \\
\text { polymerization } \\
\text { degree }\end{array}$ & $\begin{array}{c}\text { Calculated } \\
\text { molecular } \\
\text { weight of the } \\
\text { monoisotopic } \\
\text { fraction (Da) }\end{array}$ \\
\hline 1 & 4229.00 & 42 & 4228.9 \\
2 & 4328.03 & 43 & 4327.9 \\
3 & 4427.08 & 44 & 4427.0 \\
4 & 4526.13 & 45 & 4526.1 \\
5 & 4625.12 & 46 & 4625.1 \\
6 & 4724.11 & 47 & 4724.2 \\
\hline
\end{tabular}

(i) Peak numbers are assigned in Figure 2 (a)

Table 2. Mass values of monoisotopic fraction in the selected peaks of MALDI-TOF MS

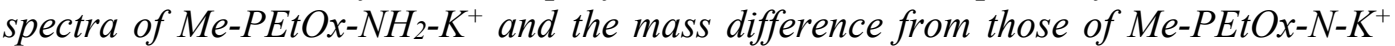
with corresponding peak number.

\begin{tabular}{ccccc}
\hline Peak number ${ }^{(\mathrm{i})}$ & $\begin{array}{c}\text { Measured mass } \\
\text { value of the } \\
\text { monoisotopic } \\
\text { fraction (m/z) }\end{array}$ & $\begin{array}{c}\text { Calculated } \\
\text { polymerization } \\
\text { degree }\end{array}$ & $\begin{array}{c}\text { Calculated } \\
\text { molecular } \\
\text { weight of the } \\
\text { monoisotopic } \\
\text { fraction (Da) }\end{array}$ & $\begin{array}{c}\text { Difference in } \\
\text { mass value from } \\
\text { corresponding } \\
\text { peak in MS of } \\
\text { Me-PEtOx- } N-K^{+} \\
(\mathrm{m} / \mathrm{z})\end{array}$ \\
\hline 1 & 4230.97 & 42 & 4230.9 & 1.97 \\
2 & 4330.02 & 43 & 4329.9 & 1.99 \\
3 & 4429.07 & 44 & 4429.0 & 1.99 \\
4 & 4528.10 & 45 & 4528.1 & 1.97 \\
5 & 4627.13 & 46 & 4627.1 & 2.01 \\
6 & 4726.14 & 47 & 4726.2 & 2.03 \\
\hline
\end{tabular}

(i) Peak numbers are assigned in Figure 2 (b) 


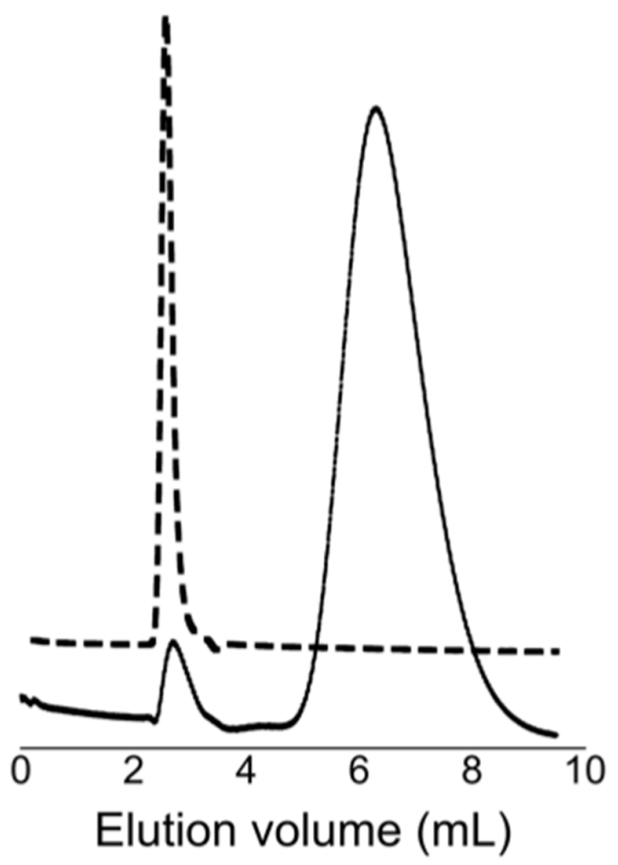

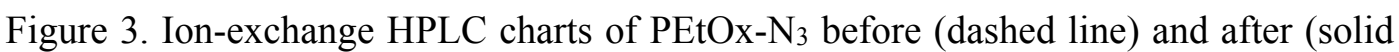
line) the Staudinger reaction for amino conversion.

\subsection{Optimized reaction time to obtain $\mathrm{PEtOx}-\mathrm{NH}_{2}$}

We further optimized the reaction time necessary for each step in Scheme 1 aiming to reduce the overall time required to obtain the amino-functionalized PEtOx. As it is difficult to directly determine the azido-functionality of PEtOx end-capped with $\mathrm{NaN}_{3}$ after the first reaction step, we alternatively determined the amino-functionality of the final product by ion-exchange HPLC method after subjecting the product of the first 
reaction step in Scheme 1 to the Staudinger reaction. Here, the reactant of EtOx polymerization with the methyl $p$-tosylate initiator was treated with $\mathrm{NaN}_{3}$ for 24 hours, assuming this much time to be sufficient for achieving maximal $\omega$-end capping of PEtOx with the azido group. The isolated product was then further reacted with TPP to obtain the amino-functionalized final product by taking several reaction times. As shown in Figure 4a, the amino-functionality of $96 \%$ has already been obtained in 3 hours for reaction with TPP, and no further increment was observed even taking longer reaction time. This result, thus, revealed that 3 hours was enough for completion of the Staudinger reaction to quantitatively convert $\mathrm{PEtOx}-\mathrm{N}_{3}$ to $\mathrm{PEtOx}-\mathrm{NH}_{2}$.

The time to complete $\omega$-end capping of the growing chain end of PEtOx with the azido group, was estimated by measuring the amino-functionality of the obtained PEtOx-NH2, using the Staudinger reaction. Here, a series of PEtOx-N 3 was prepared by varying the reaction time for $\omega$-end capping with the azido group, and was subjected to reaction with TPP to produce PEtOx- $\mathrm{NH}_{2}$. The time for the latter was set to 3 hours, based on the result demonstrated in Figure 4a. Evaluation by ion-exchange HPLC revealed that the amino-functionality of the final product has already reached $96 \%$ even 
after a reaction time of only 1 hour (Figure $4 \mathrm{~b}$ ). This result indicated that $\omega$-end capping of the growing chain end of PEtOx with $\mathrm{NaN}_{3}$ is completed within 1 hour.

Consequently, the procedure given in Scheme 1 allowed for quantitative introduction of the primary amino group at the distal chain end of PEtOx within several hours. This is clearly an advantageous procedure compared to previously reported methods, requiring several reaction steps with longer reaction times. Furthermore, the finding that the $\omega$-azido capping of the growing chain end of PEtOx can be completed within 1 hour is practically appealing, because a much longer reaction time of 24 hours had generally been adopted for this capping reaction, due to a lack of detailed kinetics $[25,26]$.

The utility of this procedure was further demonstrated through the successful application of a one-pot synthesis. PEtOx with $86 \%$ amino functionality was directly obtained from the polymerization mixture without changing the reaction vessel by 1 hour reaction for the azido termination and subsequent 3 hours reaction for the azido-to-amino conversion (Figure S3). Note that the amino-functionality obtained by the one-pot synthesis was not as high as that obtained by two-pot synthesis. As the first 
step reaction in the one-pot synthesis should proceed in a similar way to the two-pot synthesis, the different condition for the second step reaction from the two-pot synthesis, such as unchanged solvent of acetonitrile throughout the reaction and the presence of residues from the first reaction, might affect negatively to the azide-amine conversion reaction. Nevertheless, the obtained amino functionality was comparable with that obtained using conventional procedures $(\sim 89 \%)$, which require 24 hours for phthalimide introduction and another 24 hours for conversion to the amino group by hydrazine treatment $[22,23]$, appealing a facile handling of this one-pot synthetic procedure.
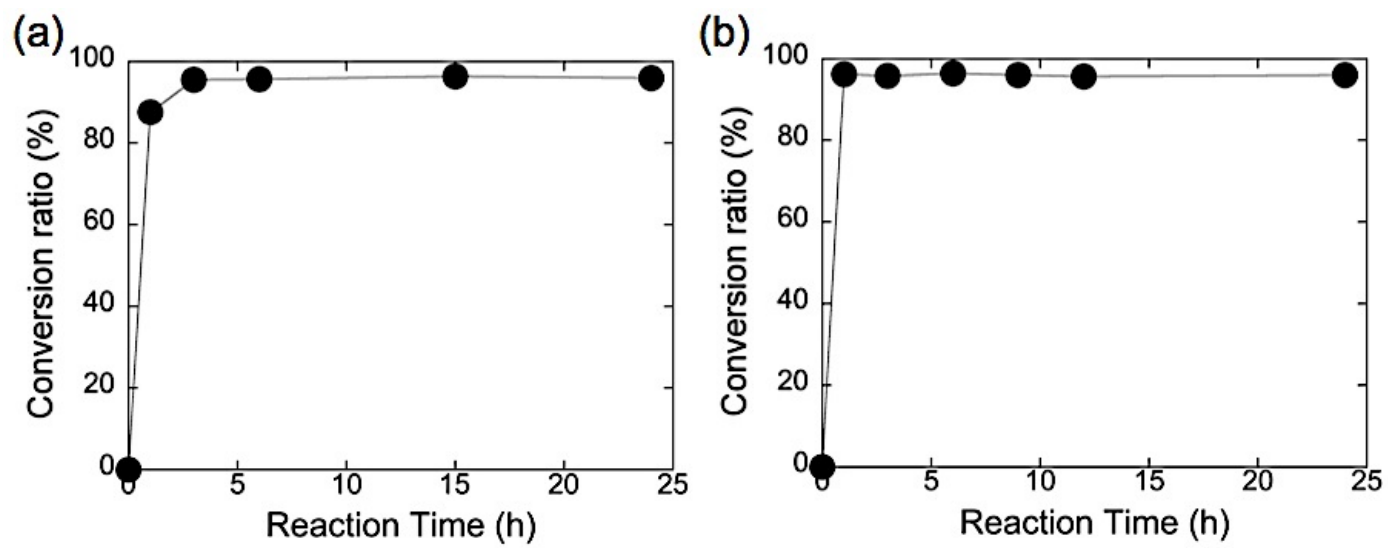

Figure 4. Conversion ratio of the $\omega$-end of PEtOx into an amino group over time. (a)

Conversion ratio vs. the reaction time with TPP for the sample pre-reacted with $\mathrm{NaN}_{3}$ for 24 hours. (b) Conversion ratio vs. the reaction time with $\mathrm{NaN}_{3}$ for the sample post-reacted with TPP for 3 hours. 


\subsection{Synthesis of PEtOx-b-PLys(TFA) from PEtOx- $\mathrm{NH}_{2}$}

As a demonstration of the utility of the amino group introduced into the $\omega$-chain end of

POxs, a ring-opening polymerization of the $\alpha$-amino acid NCA was carried out by using

synthesized $\mathrm{PEtOx}-\mathrm{NH}_{2}$ with $96 \%$ functionality as a macroinitiator, to obtain a

PEtOx-poly(amino acid) block copolymer, which may have a feasibility in drug

delivery applications. Successful block copolymerization of L-Lys(TFA)-NCA was

confirmed from the SEC profile, as shown in Figure 5. The peak corresponding to the

original PEtOx-NH2 (shown as the dashed line in Figure 5a) almost disappeared after

NCA polymerization, and a new peak was observed with a unimodal molecular weight

distribution in the higher molecular weight region with a shorter elution time (shown as

solid line in Figure 5a). Of note, a small fraction appearing in the SEC profile of block

copolymer around the region corresponding to the macroinitiator was most likely

ascribed to the non-amino functionalized PEtOx because the area ratio of this fraction in

the whole SEC profile (3\%) was comparable to the percentage of the non-aminated

fraction in the amino-functionalized PEtOx sample (4\%), as determined from the 
ion-exchange HPLC. This indicates that essentially all the fraction of the amino-functionalized PEtOx can initiate the NCA polymerization.

The ${ }^{1} \mathrm{H}-\mathrm{NMR}$ spectrum of the product clearly shows signals corresponding to the Lys(TFA) unit (signals "e" in Figure 5(b)), and accordingly, by comparing the intensity of the signals assigned to PEtOx (signal "c" in Figure 5(b)), the degree of polymerization of Lys(TFA) was calculated to be 74. PEtOx- $b$-PLys may serve as a novel platform material for gene carriers, considering that a similar block copolymer, PEG- $b$-PLys, is a well-studied gene carrier [19,20, 32,33].

(a)

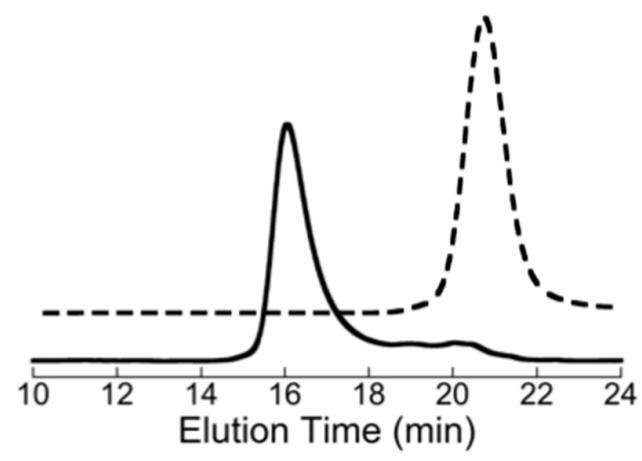

(b)

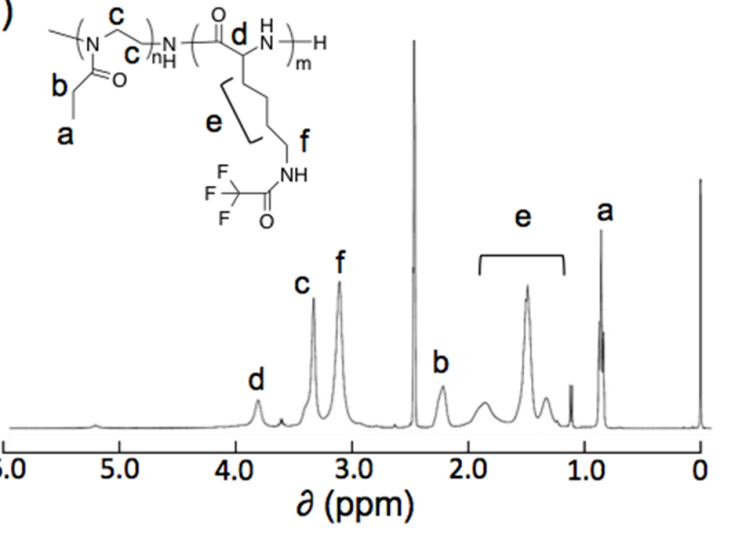

Figure 5. Characterization of PEtOx-b-PLys(TFA). (a) SEC charts for block copolymer after Lys(TFA)-NCA polymerization (solid line) and the original $\mathrm{PEtOx}-\mathrm{NH}_{2}$ before NCA polymerization (dashed line). (b) ${ }^{1} \mathrm{H}-\mathrm{NMR}$ spectrum of the synthesized block copolymer measured in $\mathrm{d}_{6}-\mathrm{DMSO}$. 


\section{Conclusion}

In this study, we established a novel procedure to introduce a primary amino group at the distal chain end of POxs by azido termination of oxazoline polymerization with $\mathrm{NaN}_{3}$, followed by azide-amine conversion by the Staudinger reaction with TPP. A detailed study of each reaction step by varying the reaction time verified that the reaction could be completed within several hours to introduce $\mathrm{NH}_{2}$ group to the synthesized polyoxazolines with $96 \%$ functionality, thus, proving this procedure to be facile and quantitative. Notably, the procedure is applicable to one-pot synthesis, yielding a product with $86 \%$ functionality. Thus, it may have a versatile application in developing POx-based functionality materials, particularly in biomedical fields.

\section{References}

1. R. Hoogenboom, Poly(2-oxazoline)s: A polymer class with numerous potential applications. Angew. Chem. Int. Ed. 48 (2009) 7978-7994.

2. O. Sedlacek, B. D. Monnery, S. K. Filippov, R. Hoogenboom, M. Hruby, Poly(2-Oxazoline)s - Are they more advantageous for biomedical applications than other polymers? Macromol. Rapid Commun. 33 (2012) 1648-1662.

3. E. Rossegger, V. Schenk, F. Wiesbrock, Design strategies for functionalized poly(2-oxazoline)s and derived materials. Polymers 5 (2013) 956-1011. 
4. K. Knop, R. Hoogenboom, D. Fischer, U. S. Schubert, Poly(ethylene glycol) in drug delivery: Pros and cons as well as potential alternatives. Angew. Chem. Int. Ed. 49 (2010) 6288-6308.

5. C. Diab, Y. Akiyama, K. Kataoka, F.M. Winnik, Microcalorimetric study of the temperature-induced phase separation in aqueous solutions of poly(2-isopropyl-2-oxazoline). Macromolecules 37 (2004) 2556-2562.

6. J.-S. Park, K. Kataoka, Comprehensive and accurate control of thermosensitivity of poly(2-alkyl-2-oxazoline)s via well-defined gradient or random copolymerization. Macromolecules 40 (2007) 3599-3609.

7. M. Hartlieb, D. Pretzel, K. Kempe, C. Fritzsche, R. M. Paulus, M.Gottschaldt, U. S. Schubert, Cationic poly(2-oxazoline) hydrogels for reversible DNA binding. Soft Matter 9 (2013) 4693-4704.

8. K. Lava, B. Verbraeken, R. Hoogenboom, Poly(2-oxazoline)s and click chemistry: A versatile toolbox toward multi-functional polymers. European Polym. J. 65 (2015) 98-111.

9. S. Cesana, A. Kurek, M. A. Baur, J. Auernheimer, O. Nuyken, Polymer-bound thiol groups on poly(2-oxazoline)s. Macromol. Rapid Commun. 28 (2007) 608-615.

10. A. Takasu, H. Kojima. Synthesis and ring-opening polymerizations of novel S-glycooxazolines, J. Polym. Sci. A Polym. Chem. 48 (2010) 5953-5960.

11. R. Konradi, C. Acikgoz, M. Textor, Polyoxazoline for nonfouling surface coatings A direct comparison to the gold standard PEG. Macromol. Rapid Commun. 33 (2012) 1663-1676.

12. B. Pidhatika, M. Rodenstein, Y. Chen, E. Rakhmatullina, A. Muhlebach, C. Acikgoz, T. Marcus, R. Konradi, Comparative stability studies of Poly(2-methyl-2-oxazoline) and Poly(ethylene glycol) brush coatings. Biointerphases 7:1 (2012).

13. A. Mero, G. Pasut, Via L. Dalla, M. W. Fijten, U. S. Schubert, R. Hoogenboom, F. M. Veronese, Synthesis and characterization of poly(2-ethyl-2-oxazoline)-conjugates with proteins and drugs: suitable alternative to PEG-conjugate? J. Controlled Release 2 (2008) 87-95.

14. J. Tong, R. Luxenhofer, X. Yi, R. Jordan, A. V. Kabanov, Protein modification with amphiphilic block copoly(2-oxazoline) as a new platform for enhanced cellular delivery. Mol. Pharm. 7 (2010) 984-992. 
15. J. F. Nawroth, J. R. Mcdaniel, A. Chilkoti, R. Jordan, R. Luxenhofer, Maleimide-functionalized poly(2-oxazoline)s and their conjugation to elastin-like polypeptides. Macromolecular Bioscience 16 (2016) 322-333.

16. B. L. Farrugia, K. Kempe, U. S. Schubert, R. Hoogenboom, T. R. Dargaville, Poly(2-oxazoline) hydrogels for controlled fibroblast attachment.

Biomacromolecules 14 (2013) 2724-2732.

17. C. Legros, A.-L. Wirotius, M.-C. De Pauw-Gillet, K. C. Tam, D. Taton, S. Lecommandoux, Poly(2-oxazoline)-based nanogels as biocompatible pseudopolypeptide nanoparticles. Biomacromolecules 16 (2015) 183-191.

18. J. Li, Y. Zhou, C. Li, D. Wang, Y. Gao, C. Zhang, L. Zhao, Y. Li, Y. Liu, X. Li, Poly(2-ethyl-2-oxazoline)-doxorubicin conjugate-based dual endosomal pH-sensitive micelles with enhanced antitumor efficacy. Bioconjugate Chem. 26 (2015) 110-119.

19. S. Osawa, K. Osada, S. Hiki, A. Dirisala, T. Ishii, K. Kataoka, Polyplex micelles with double-protective compartments of hydrophilic shell and thermo-switchable palisade of poly(oxazoline)-based block copolymers for promoted gene transfection. Biomacromolecules 17 (2016) 246-255.

20. K. Osada, K. Kataoka, Drug and gene delivery based on supramolecular assembly of PEG-polypeptide hybrid block copolymers. Adv. Polym. Sci. 202 (2006) 113-153.

21. H. Cabral, N. Nishiyama, K. Kataoka, Supramolecular nanodevices: From design validation to theranostic nanomedicine. Acc. Chem. Res. 44 (2011) 999-1008.

22. J-S. Park, Y. Akiyama, F. M. Winnik, K. Kataoka, Versatile synthesis of end-functionalized thermosensitive poly(2-isopropyl-2-oxazolines), Macromolecules 37 (2004) 6786-6792.

23. L. Tauhardt, M. Frant, D. Pretzel, M. Hartlieb, C. Buecher, G. Hildebrand, B. Schroeter, C. Weber, K. Kempe, M. Gottschaldt, K. Liefeithc, U. S. Schubert, Amine end-functionalized poly(2-ethyl-2-oxazoline) as promising coating material for antifouling applications. J. Mater. Chem. B 2 (2014) 4883-4893.

24. M. Meyer, H. Schlaad, Poly(2-isopropyl-2-oxazoline)-poly(L-glutamate) block copolymers through ammonium-mediated NCA polymerization. Macromolecules 39 (2006) 3967-3970. 
25. G. Volet, T. X. Lav, J. Babinot, C. Amiel, Click-Chemistry: An Alternative Way to functionalize poly(2-methyl-2-oxazoline). Macromol. Chem. Phys. 212 (2011) 118-124.

26. P. Lemechko, E. Renard, G. Volet, C. S. Colin, J. Guezennec, V. Langlois, Functionalized oligoesters from poly(3-hydroxyalkanoate)s containing reactive end group for click chemistry: Application to novel copolymer synthesis with poly(2-methyl-2-oxazoline), React. and Func. Polym. 72 (2012) 160-167.

27. H. Staudinger, J. Meyer, Uber neue organische Phosphorverbindungen 111. Phosphinmethylenderivate und Phosphinimine. Helv. Acta. 2 (1919) 635-646.

28. F. L. Lin, H. M. Hoyt, H. van Halbeek, R. G. Bergman, C. R. Bertozzi, Mechanistic investigation of the Staudinger ligation. J. Am. Chem. Soc. 127 (2005) 2686-2695.

29. J. R. Hernandez, H. A. Klok, Synthesis and ring-opening (co)polymerization of L-lysine $N$-carboxyanhydrides containing labile side-chain protective groups. $J$. Polym. Sci. Part A Polym. Chem. 41 (2003) 1167-1187.

30. John Wiley \& Sons, Reactions, Mechanisms, and Structure, 5th ed. p 1412.

31. S. Hiki, K. Kataoka, A facile synthesis of azido-terminated heterobifunctional poly(ethylene glycol)s for "Click" conjugation. Bioconjugate Chem. 18 (2007) 2191-2196.

32. S. Katayose, and K. Kataoka, Water-soluble polyion complex associates of DNA and poly(ethylene glycol)-poly(L-lysine) block copolymer. Bioconjugate Chem. 8 (1997) 702-707.

33. A. Dirisala, K. Osada, Q. Chen, T. A. Tockary, K. Machitani, S. Osawa, X. Liu, T. Ishii, K. Miyata, M. Oba, S. Uchida, K. Itaka, K.Kataoka, Optimized rod length of polyplex micelles for maximizing transfection efficiency and their performance in systemic gene therapy against stroma-rich pancreatic tumors. Biomaterials 35 (2014) 5359-5368.

\section{Acknowledgement}

This work was financially supported by Center of Innovation (COI) program 
and "Precursory Research for Embryonic Science and Technology" (PRESTO) in "Molecular Technology and Creation of New Functions" from the Japan Science and Technology Corporation (JST), and the Japan Society for the Promotion of Science (JSPS) through Specially Promoted Research Program, and Core to Core Program for A. Advanced Research Networks. 
